\title{
Johannes Kepler on Christmas
}

\author{
Kepler's interpretation of the supernova of 1604, De Stella Nova, interwove the science of astronomy with \\ astrology and theology in an attempt to determine the correct birthdate of Jesus, explains Martin Kemp.
}

When was Jesus born? On 25 December in the year that starts the Christian calendar, is what wide belief would have it. Greek and Russian Orthodox churches retain the date of 7 January in accordance with the old Julian calendar, rather than the Gregorian revision that was introduced in 1582 .

Internal evidence from the Bible rules out the designated year because Herod the Great, who was responsible for the massacre of the innocents, from which the infant Jesus escaped, is known to have died in $4 \mathrm{BC}$. This is one reason why the now-obscure Polish scholar, Laurence Suslyga, argued in his 1605 thesis on the birth and death of Christ that Jesus was actually born in 5 BC.

Suslyga's tract unexpectedly gained a significance beyond the specialist realm of biblical chronology. The circumstances are best explained by the great German astronomer, Johannes Kepler: "I found for sale at Graz a small book by Laurence Suslyga of Poland," with which Kepler agreed that at least "four years must be added to the Epoch of Christianity now in use". Suslyga provided Kepler with the necessary licence to correlate the dramatic appearance of a 'new star' in 1604 with the act of stellar navigation performed by the biblical Magi when they journeyed to see the Christ child in Bethlehem.

In 1604, astronomers keenly awaited the shift of the conjunction of Jupiter and Saturn into the 'Fiery Trigon' of the zodiac (Sagittarius, Aries and Leo), which initiated a new cycle of conjunctions in the Trigon - an event that was calculated to recur about every 800 years. Mars also moved close to Saturn and then Jupiter, thus outlining a triangular array that was of great interest to astrologers.

During his time in Prague as mathematician to the Holy Roman Emperor Rudolf II, Kepler observed this remarkable cluster of planets and the new star on 17 October 1604, when the clouds over the city finally lifted. The star burned brightly in the evening and was even visible as a morning star, located in the foot of the constellation of the Serpent Bearer, or Ophiuchus (pictured). He made his last observation of the star a year later, after

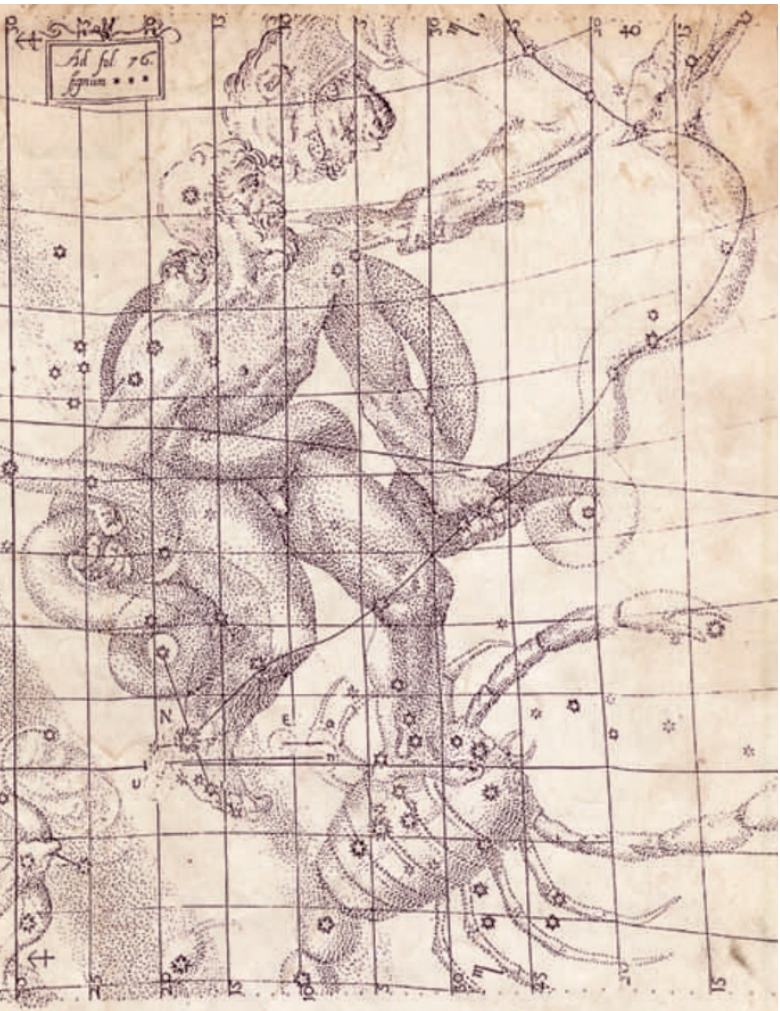

rtwork from De Stella Nova shows the constellation Ophiuchus with 1604 supernova ( $N$; in the foot, lower left) observed by Kepler.

Kepler's treatise can be acclaimed as a work of science in the modern sense. For instance, he uses the lack of diurnal parallax from the new star - that is, the inability to measure its distance using the angle at two different points on Earth's surface - to infer that it resided in the sphere of the fixed stars. This provided further evidence that the sphere was not as immutable as the ancients had assumed. But De Stella Nova, as its extended title declares, explicitly involved metaphysics and astrology, and it climaxed in an appendix on biblical chronology.

Within this, we cannot separate what we regard as the real science from the astrology and theology. For Kepler, mathematics and meaning comprised a unified whole. The great astronomer, best known for identifying the elliptical paths of the planets, became increasingly concerned in the years following publication of $D e$ Stella Nova with the construction of a 'purified' version of ancient astrological wisdom, purged of the myths that had accumulated around the zodiacal signs. He later referred to the mystic symbolism of traditional

which it faded from view as the flash from its explosion declined in intensity.

In 1606, Kepler published a pamphlet on the new star in German, while planning a more substantial account in Latin: On the New Star in the Foot of the Ophiuchus and on the Fiery Trigon that Began Anew at its Rising. A Booklet Full of Astronomical, Physical, Metaphysical, Meteorological and Astrological Disputations (beginning in Latin, De Stella Nova in Pede Serpentarii...). The second of two substantial appendices in the book dealt with the year of Christ's birth "in the light of the new pronouncements of Laurentius Suslyga".

In a series of arguments that even he admitted were hard to follow, Kepler claimed that the star followed by the Magi was the equivalent of the stella nova of 1604-5, and that it had arisen during a series of related planetary conjunctions in the years 7-5 BC - which he took to cover the period of Christ's conception and the Magi's journey to Bethlehem, as recounted in Matthew 2:9-10. astrology as "filthy mud" from which "one can glean even an occasional escargot, oysters or an eel for one's nutrition".

Kepler gained his "nutrition" from the mathematical ratios of the 'aspects' between the planets. He defined an aspect as "a geometrical construction [of an angle] between light beams of two planets here on Earth". The ratios were integral to the celestial geometry that manifests the mathematical "music" of the heavens. He explained that earthly nature cannot help but respond to the dictates of heavenly harmonies, and said that nature is affected by an aspect "just as a farmer is moved by music to dance".

De Stella Nova serves to remind us that it was not possible in the era of Kepler and Galileo to pursue astronomy in such a way that the mathematical study of the heavenly bodies was divorced from the theology of a heaven inhabited by God.

Martin Kemp is emeritus professor in history of art at the University of Oxford, Oxford, UK. 